Service social

\title{
La Commission Rochon : enjeux pour le service social, les services sociaux et la société.
}

\section{Élaine Carey-Bélanger}

Volume 34, numéro 2-3, 1985

L'organisation communautaire

URI : https://id.erudit.org/iderudit/706267ar

DOI : https://doi.org/10.7202/706267ar

Aller au sommaire du numéro

Éditeur(s)

École de service social de l'Université Laval

ISSN

1708-1734 (numérique)

Découvrir la revue

Citer ce document

Carey-Bélanger, É. (1985). La Commission Rochon : enjeux pour le service social, les services sociaux et la société. Service social, 34(2-3), 202-205. https://doi.org/10.7202/706267ar d'utilisation que vous pouvez consulter en ligne. 


\section{ÉDITORIAL}

\section{La Commission Rochon : enjeux pour le service social, les services sociaux et la société}

\section{Élaine Carey-Bélanger}

Créée le 20 juin 1985 par le Gouvernement du Québec, au moment où le Parti québécois était au pouvoir, la Commission sur la santé et les services sociaux (mieux connue sous le nom de son président, Jean Rochon) est vite apparue comme le précurseur de nouvelles réformes. En ce sens, elle suscite à la fois de l'intérêt, des réactions et des attentes.

Cette Commission est aussi la troisième à prendre place dans une longue lignée de réformes, depuis la Révolution tranquille et de concert avec la modernisation de l'État québécois. En effet, en 1963, le Comité d'étude sur l'assistance publique (Commission Boucher) avait mis de l'avant, pour la première fois, la responsabilité prépondérante du secteur public dans l'organisation, la coordination et la distribution des services d'aide financière, et le besoin d'une politique articulée de services de santé et de services sociaux.

De plus, de 1966 à 1971, la Commission d'enquête sur la santé et le bien-être social (Commission Castonguay-Nepveu) a provoqué une réorganisation quasi totale, les services de santé et les services sociaux étant intégrés à l'intérieur du Ministère des affaires sociales. On y mettait en application les quatre postulats de base de cette réforme: l'accessibilité, l'universalité, l'efficacité et la participation.

\section{Contexte et mandat}

Plusieurs problèmes surgissant au fil des ans, les individus et les collectivités ressentent une inquiétude de plus en plus grande à l'égard 
des institutions et du réseau de services; la bureaucratisation a comme résultat une parcellisation des besoins, une insensibilité par rapport aux besoins nouveaux et une inflexibilité face aux changements.

Une dépendance plus grande envers l'État semble s'accompagner d'une perte d'identité individuelle et collective : il y a un déclin de la cohésion sociale, qui est fondée sur les forces vives de la société. Officiellement, le Québec a un système de santé et de services sociaux très avancé; mais les problèmes en présence, les coûts toujours plus élevés de son maintien et la présente conjoncture fiscale et sociale ont provoqué une remise en question et la création de la nouvelle Commission d'enquête.

Dans un premier temps, celle-ci avait comme mandat d'examiner le système de soins de santé et les services sociaux connexes, leur mode de financement et leur fonctionnement. On visait à déterminer les types de services que le Québec pouvait se permettre de payer et à établir les priorités à être respectées par les politiciens, pour en arriver à des choix collectifs en matière de services. À la fin de janvier 1986, après un changement de gouvernement, le mandat, la composition et l'échéancier de la Commission étaient modifiés. L'ensemble des services sociaux est dorénavant intégré dans le mandat ; le nombre de commissaires est réduit et inclut maintenant un travailleur social ; le rapport final est attendu pour le 30 septembre 1987, donc trois mois plus tôt que l'échéancier initial.

À travers un processus de consultation étendu, la Commission tentera de définir les problématiques et les enjeux, ainsi que les scénarios de solutions.

Au départ, il faut convenir que la conjoncture socio-politique et économique présente est très différente de celle des années 1960 et 1970. L'État-Providence, vu alors comme essentiel, est présentement remis en question. Dans les domaines de la santé et des services sociaux, nous décelons une nouvelle philosophie de retrait de la part de l'État; d'ailleurs, cette prise de position fait partie d'une vague présente, non seulement au Québec, mais dans toute l'Amérique du Nord et dans les pays d'Europe occidentale en général. II peut en découler une remise en question de l'universalité des prestations et des services ainsi que de droits acquis, tout comme de plus grandes inégalités sociales peuvent résulter du gel ou des coupures dans les budgets des services sociaux. Au niveau de la Commission, on reconnaît déjà l'incapacité du système de maintenir la précédente réforme en termes financiers; et, dans plusieurs des mémoires déjà présentés, on évoque l'hypothèse d'un virage radical allant jusqu'à la privatisation de certains services. Nous nous retrouvons aussi, globalement, dans une 
situation de crise économique qui génère des problèmes plus nombreux et plus aigus à être considérés par les services sociaux, en même temps que les ressources de ceux-ci sont de plus en plus limitées.

\section{Principaux enjeux}

Sans identifier l'ensemble des enjeux présents dans un tel débat, nous avons voulu en cerner quelques-uns qui touchent le domaine social.

1. Un concept global de la santé. Comment définir la santé et la maladie? Ou plutôt, comment définir l'état de bien-être qu'une société veut pour ses membres? La santé, vue sous l'angle d'un processus physique et mental dont la responsabilité incombe à chaque individu et à son milieu de vie, favorise la prise en charge du processus en tenant compte des conditions objectives de l'existence et des composantes structurelles, socio-économiques et psychosociales qui nuisent ou supportent un état de bien-être. Ce concept permettrait une meilleure clarification des notions de services préventifs et curatifs, et favoriserait une approche basée sur la force des individus et une attaque concertée contre les problèmes communs qui compromettent la santé.

2. Coûts sociaux ou coûts des services sociaux. Dans les discussions actuelles, on fait fréquemment référence aux coûts sociaux dans leur globalité. Il est important de distinguer entre les coûts spécifiques des secteurs santé, sécurité du revenu et services sociaux. Pour en arriver à déterminer si ces derniers sont dispendieux ou non, il faut tenir compte des besoins présents, de la disponibilité et de la localisation des différentes ressources, comme de la pertinence et de la qualité des services.

3. Travail social et contradictions de la société. De par sa fonction, le travail social permet de rendre compte de la nature et de l'étendue des problèmes, de contribuer au soulagement des tensions et de défendre les intérêts des groupes et des populations défavorisés, marginalisés et vulnérables. Tout en dénonçant les contradictions du système et les effets directs ou indirects d'une dynamique conflictuelle des rapports sociaux, il se doit d'apporter des précisions sur les actions à entreprendre et sur les conditions de pratique nécessaires pour les réaliser.

Si ce double volet n'est pas mis en valeur, il risque de s'ensuivre une fragmentation et une déqualification de la pratique sociale. II faut que s'affirme ce double rôle : dénoncer les conditions qui poussent à la marginalisation de certaines parties de la population, et apporter 
une aide dans les situations précises pour redonner à celles-ci leur dignité et pour dégager leurs capacités propres de résolution de problèmes; ce double rôle constitue la véritable expertise du travail social.

4. Dualisation du social. La notion de services sociaux fait à la fois référence à un système institutionnel et à un système alternatif ou hors réseau. Les deux ont d'ailleurs développé plusieurs pratiques innovatrices au cours des dernières années. Une dimension importante touche actuellement la concertation entre les différents acteurs en présence, et la recherche d'une cohérence et d'une clarification des rôles entre les bénévoles, les groupes autonomes, les ressources alternatives, et les services du système institutionnel, afin de développer une collaboration plutôt qu'une rivalité.

5. Participation et implication. Au cours des dernières années, des transferts de ressources ont été effectués, sans consultation des populations directement impliquées, sans bilan ni évaluation des impacts sociaux. Ceci semble assez loin de la participation valorisée par la réforme Castonguay-Nepveu, participation qui reste très importante au moment de la nouvelle Commission, tant dans son processus que dans les postulats qu'elle avance.

Par leur rôle et par leur position en égard à plusieurs clientèlescibles, les travailleurs sociaux peuvent être des interlocuteurs privilégiés pour reconnaître les conditions de vie et les besoins de la population. Mais d'abord, il est plus que souhaitable que le type de recherche privilégié, le choix des acteurs écoutés et le type de planification de la Commission laissent une large place à une participation de la population.

Nous n'avons pas indiqué, ici, l'ensemble des enjeux liés à la Commission sur la santé et les services sociaux. Ainsi, on aurait pu également souligner, en se référant à des mémoires déjà présentés ou à de récents énoncés de programmes gouvernementaux, le débat sur la question de la privatisation et de l'universalité des services, que l'on peut aussi relier à la question de la responsabilité sociale de l'État eu égard à l'accessibilité des services.

Nous l'avons déjà dit, la Commission Rochon soulève beaucoup d'attentes et beaucoup d'espoir. Il y a une mobilisation évidente dans plusieurs milieux et nous croyons que c'est de bon droit, car les propositions qui vont en découler risquent d'influencer les services sociaux de la prochaine décennie, sinon davantage. 